\title{
Does Exogenously Applied Gallic Acid Regulate the Enzymatic and Non- Enzymatic Antioxidants in Wheat Roots Exposed to Cadmium Stress?
}

\author{
Ceyda Ozfidan Konakci* \\ Necmettin Erbakan University, Faculty of Science, Department of Molecular Biology and Genetics, Konya, Turkey
}

*cozfidan@erbakan.edu.tr

Received: 17 April 2019

Accepted: 13 September 2019

DOI: $10.18466 /$ cbayarfbe. 554860

\begin{abstract}
The aim of the current study was to determine whether gallic acid (GLA) triggers the growth, osmoregulation and antioxidant system related to defense mechanisms in wheat roots to cadmium (Cd)induced oxidative stress. For this purpose, wheat plants were hydroponically grown for 21 (d) and were treated with GLA (GLA1-2; 25 and $75 \mu \mathrm{M})$, Cd stress $(\mathrm{Cd} 1-2 ; 100$ and $200 \mu \mathrm{M})$ and their combination for $7 \mathrm{~d}$. The significant reduction in growth (RGR) and osmotic potential $\left(\Psi_{\Pi}\right)$ was observed under stress. After GLA applications in response to stress, RGR, $\Psi_{\Pi}$ and proline (Pro) increased, except for $200 \mu \mathrm{M}$ Cd plus $75 \mu \mathrm{M}$ GLA. Under stress, hydrogen peroxide $\left(\mathrm{H}_{2} \mathrm{O}_{2}\right)$ was induced by the activated superoxide dismutase (SOD) activity but, NADPH oxidase (NOX) had no contribution on the accumulation of $\mathrm{H}_{2} \mathrm{O}_{2}$. Despite of the increased catalase (CAT) and glutathione reductase (GR), $\mathrm{H}_{2} \mathrm{O}_{2}$ did not eliminate and then lipid peroxidation (TBARS content) was induced with the decreased scavenging capacity of hydroxyl radical $\left(\mathrm{OH}^{*}\right)$ under stress. Besides, to remove of $\mathrm{H}_{2} \mathrm{O}_{2}$ content produced by SOD, $\mathrm{H}_{2} \mathrm{O}_{2}$ could effectively scavenge through CAT activity in combination form of GLA and Cd. On the other hand, GLA did not induce the enzymes and non-enzymes related to Asada-Halliwell cycle (ascorbate peroxidase (APX), GR, dehydroascorbate reductase (DHAR), monodehydroascorbate reductase (MDHAR), reduced and oxidized contents of glutathione (GSH and GSSG contents). Under high Cd concentration, GLA2 could not eliminate $\mathrm{H}_{2} \mathrm{O}_{2}$ content because of increased NOX activity and then in this group (Cd2+GLA2) the scavenging capacity of $\mathrm{OH}^{\bullet}$ did not change and TBARS content increased.
\end{abstract}

Keywords: Antioxidant enzymes, Asada-Halliwell pathway, Cadmium, Gallic acid, Lipid peroxidation.

\section{Introduction}

One of the global environmental problems is the contamination in soils due to toxic metals reached the high levels [1]. Cadmium (Cd) accumulates in soils and irrigation water. Plants have especially suffered from the negative effects of $\mathrm{Cd}$ on organelles such as chloroplast and mitochondria on which basic metabolic events occur. $\mathrm{Cd}$ causes degradation of chlorophyll biosynthesis and structure, inhibition of enzyme activity, reduction in $\mathrm{CO}_{2}$ assimilation levels and photosynthetic capacity, disruption of basic elements such as magnesium, calcium and potassium [2]. Besides, Cd stress-mediated radicals (Reactive oxygen species; ROS) triggers the oxidation process on significant molecules, protein and nucleic acids, and this situation results in oxidative stress in plants [1]. Plants have evolved significant strategies against stress conditions such as efflux or compartmentalization of $\mathrm{Cd}$, the production of $\mathrm{Cd}$ phytochelatin complex and overproduction of signaling molecules [3]. A great step for tolerance in Cd-treated plants is activation of enzyme/non-enzyme antioxidants [2] such as superoxide dismutase (SOD), peroxidase
(POX), catalase (CAT), glutathione reductase (GR), ascorbate peroxidase (APX) and the contents of ascorbate (AsA) and glutathione (GSH). To the conversion of hydrogen peroxide $\left(\mathrm{H}_{2} \mathrm{O}_{2}\right)$ to non-toxic forms, the Asada-Halliwell pathway (AscorbateGlutathione Cycle) is linked to the tolerance against stress damages in plants. This cycle consists APX, GR, monodehydroascorbate reductase (MDHAR), dehydroascorbate reductase (DHAR), AsA and GSH [2].

Several studies reported that because of their antioxidant capability some phenolic compounds can scavenge the toxic ROS levels. For example, the exogenous application of salicylic acid in rice plants subjected to salt stress regulated the total activity and expression of antioxidant enzymes [4]. Another study showed that ellagic acid caused accumulation of proline (Pro) content which plays in osmoregulation via increased absorption of water uptake and scavenge of stress-induced radical content [5]. Similarly, gallic acid (GLA), counting as a phenolic compound, has antioxidant activity due to two or more hydroxyl groups in chemical structure [6]. Kamdem et al. [7] detected that the highest toxic species 
of ROS produced in Fenton reaction, hydroxyl radical, is eliminated by GLA activation. Besides, the recent reports showed that the exogenously applied GLA alleviated the oxidative damages against stress conditions such as salinity, drought and cold [8,9]. However, a detailed tolerance mechanism of GLA-applied wheat through activated antioxidant system in regulating $\mathrm{Cd}$ stress is remains unknown.

Among the cereal products, wheat (Triticum aestivum L.) is one of the main food sources for humanity. Worldwide, it occurs nearly $55 \%$ of the consumed carbohydrates globally [10]. Therefore, because of its economically importance, wheat was chosen as a plant material in the current study. It was aimed with this study as follows (i) to determinate the effect of $\mathrm{Cd}$ stress on the growth, osmolyte accumulation (Pro content), ROS content (hydrogen peroxide $\left(\mathrm{H}_{2} \mathrm{O}_{2}\right)$ content and the scavenging capacity of hydroxyl radical $\left.\left(\mathrm{OH}^{\bullet}\right)\right)$, lipid peroxidation (TBARS content) and the activities of some enzymes (SOD, CAT, POX, NOX, APX, MDHAR, GR and, DHAR and the contents of GSH and GSSG in Cd-treated wheat roots (ii) to evaluate whether these parameters are affected by GLA application under Cd stress (iii) if there is an alleviation of exogenously GLA treatments on wheat roots, is there any effect of AsA-GSH cycle in this protection and how does GLA affect the enzyme system involved in this cycle.

\section{Materials and Methods}

\subsection{Plant material}

Wheat (T. aestivum L.) was chosen as a plant material.

\subsection{Methods}

\subsubsection{Experimental Design}

Seeds of wheat (T. aestivum L.) were sterilized and were germinated on the filter paper. After germinated seedling was hydroponically grown in half strength Hoagland solution under controlled growth conditions for 21 days (d), the gallic acid concentrations (GLA1-2; 25 and 75 $\mu \mathrm{M}$, respectively) were treated only or in combination with cadmium (Cd1-2, $100 \mu \mathrm{M}$ and $200 \mu \mathrm{M}$, respectively). The treatment continued for $7 \mathrm{~d}$ and then the plants were harvested.

\subsubsection{Determination of growth, osmotic potential and proline content}

The relative growth rate (RGR), root osmotic potential $\left(\Psi_{\Pi}\right)$ and Pro content were analyzed according to Yildiztugay et al. [9].

\subsubsection{Determination of ROS accumulation}

$\mathrm{H}_{2} \mathrm{O}_{2}$ content and $\mathrm{OH}^{\bullet}$ scavenging activity were made the method suggested by Yildiztugay et al. [9]. For determination of $\mathrm{H}_{2} \mathrm{O}_{2}$ content, the leaves were homogenized in cold acetone and centrifuged. The supernatant was mixed with titanium reagent and then ammonium hydroxide was added to precipitate the titanium-peroxide complex. The reaction mixture was centrifuged. The pellet was washed with cold acetone and dissolved. The absorbance of the solution was measured at $410 \mathrm{~nm} . \mathrm{H}_{2} \mathrm{O}_{2}$ concentrations were calculated using a standard curve prepared with known concentrations of $\mathrm{H}_{2} \mathrm{O}_{2}$.

\subsubsection{Determination of lipid peroxidation}

The level of lipid peroxidation called as thiobarbituric acid reactive substances (TBARS) was detected according to Yildiztugay et al. [9].

\subsubsection{Determination of isozyme and total activity of some enzymes}

For determination of SOD isoforms and total SOD activity, the procedure was completed [8]. Analysis of CAT isozymes/activities was performed the procedure given by Yildiztugay et al. [9]. POX isozymes, total POX activity, NOX isozyme and its activity were done [8, 9]. Samples containing equal amounts of protein $(25 \mu \mathrm{g})$ for isozyme activities of SOD, CAT, POX and APX were subjected to non-denaturing polyacrylamide gel electrophoresis (PAGE). Known standard amounts of enzymes (0.5 units of SOD and 0.2 units of CAT and POX) were loaded onto gels.

\subsubsection{Enzyme/non-enzyme assays related to AsA- GSH cycle}

APX, GR, DHAR and MDHAR and the contents of GSH and GSSG were assayed the procedure given by OzfidanKonakci et al. [8] and Yildiztugay et al. [9].

\subsubsection{Statistical analysis}

The results were repeated thrice independently. The value was statistically analyzed with SPSS 20.0. Comparisons with $\mathrm{p}<0.05$ were considered significantly different. In all figures, the error bars represent standard errors of the means.

\section{Results and Discussion}

\subsection{Growth, osmotic potential and Pro content}

As shown in Figure 1a, after Cd treatments, RGR decreased gradually in wheat roots. However, this decline was prevented with GLA applications. In contrast, the adverse changes were observed in roots subjected to GLA alone or GLA+Cd compared to the control or stress conditions, respectively. Likewise, Singh et al. [11] detected that GLA-treated rice seedlings had an induction in growth. This induction might be related to the cell elongation or division of roots because of effects of benzoic acid derivatives on indole acetic acid, as suggested by Hussain et al. [12]. On the other hand, while stress caused a decline in $\Psi_{\Pi}$, GLA under stress enhanced the levels of $\Psi_{П}$ (Figure 1b), except for Cd2+GLA2. GLA alone showed any major alteration in $\Psi_{\Pi}$ compared with control group. The wheat roots with $\mathrm{Cd}$ stress did not exhibit a change in Pro content (Figure 1c). On the other hand, in response to Cd stress, GLA application increased Pro content as compared to the stress group, except $200 \mu \mathrm{M}$ Cd plus $75 \mu \mathrm{M}$ GLA. Pro 
content maintains high water content necessary for RGR [13]. In the present study, there was a positive interaction among RGR, Pro content and $\Psi_{\Pi}$.
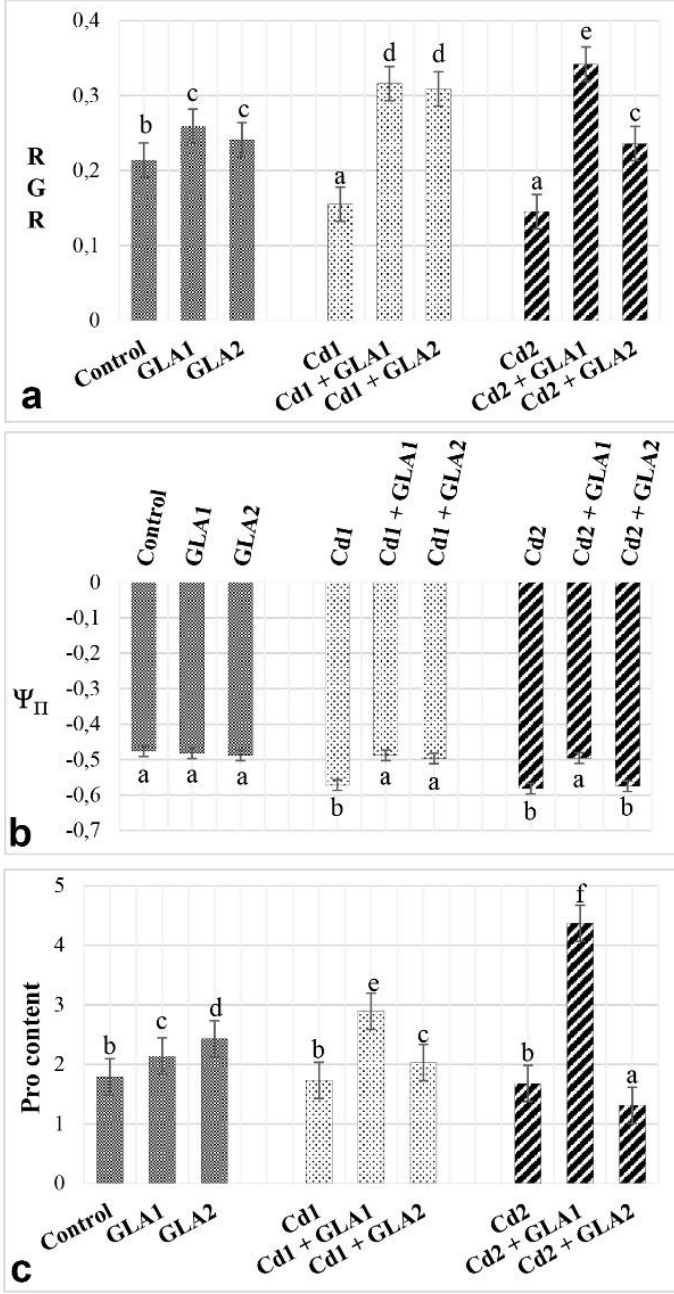

Figure 1. Relative growth rate (RGR, a), osmotic potential $\left(\Psi_{\Pi}, \mathrm{b}\right)$ and proline content (Pro, c) in wheat roots after $\mathrm{Cd}(\mathrm{Cd} 1,100$ and $\mathrm{Cd} 2,200 \mu \mathrm{M})$ and GLA (GLA1, $25 \mu \mathrm{M}$ and GLA2, $75 \mu \mathrm{M}$ ).

\subsection{ROS accumulation and lipid peroxidation}

Figure 2a reveals that $\mathrm{H}_{2} \mathrm{O}_{2}$ content of $\mathrm{Cd}$-treated wheat was higher than control group and it reached the maximum levels at $100 \mu \mathrm{M} C d$ stress (by $31.6 \%$ increase). Similar results have been reported by El-Soud et al. [5]. On the other hand, except for Cd2 + GLA2, GLA application led to a markedly decrease in $\mathrm{H}_{2} \mathrm{O}_{2}$ content induced by stress. Our data is in accord with the findings obtained by Yildiztugay et al. [9]. GLA application alone showed no statically increase in $\mathrm{H}_{2} \mathrm{O}_{2}$ content. As shown in Figure 2b, there was a decrement in the scavenging capacity of $\mathrm{OH}^{*}$ in $\mathrm{Cd}$-treated roots. However, when GLA and stress were applied together, the scavenging activity increased but not in Cd2+GLA2treated wheat. Also, the response did not detect between control and GLA alone on the scavenging activity. In accordance with $\mathrm{H}_{2} \mathrm{O}_{2}$ content, the presence of $\mathrm{Cd}$ resulted in the significant increases in lipid peroxidation (TBARS content) and a maximum response was at 200 $\mu \mathrm{M} \mathrm{Cd}(83.8 \%$ increase) (Figure 2c). It was suggested by Guo et al. [14] that one of the great causes of lipid peroxidation is $\mathrm{Cd}$ treatments. In the present study, this effect caused by stress was prevented though GLA treatments except for Cd2+GLA2. Also, GLA application created any affect in TBARS content. Similar responses had been detected by Ozfidan-Konakci et al. [8]. There was a closely relation between the disruption of scavenging activity and TBARS content.

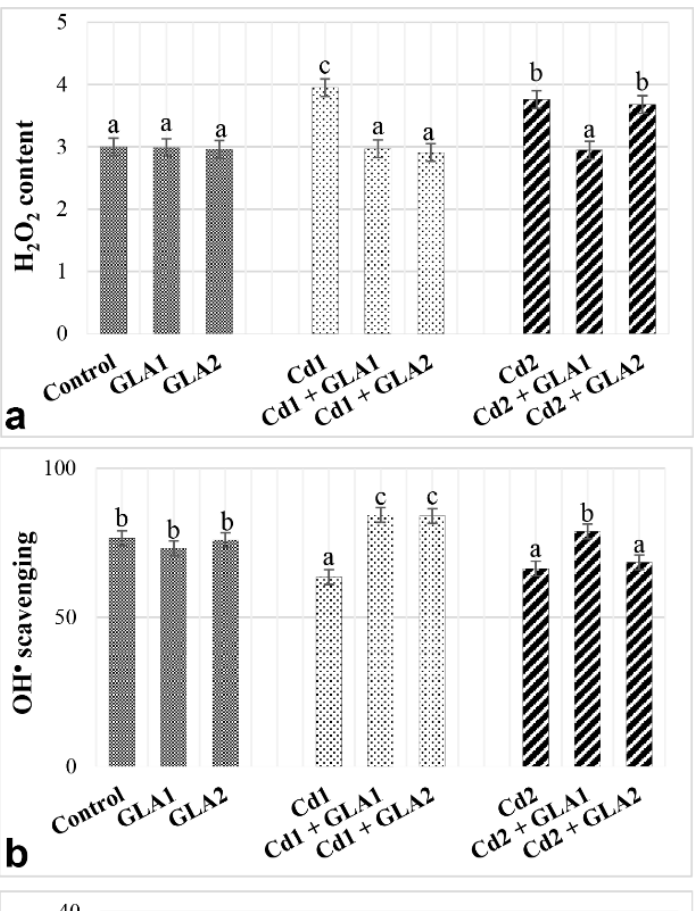

40

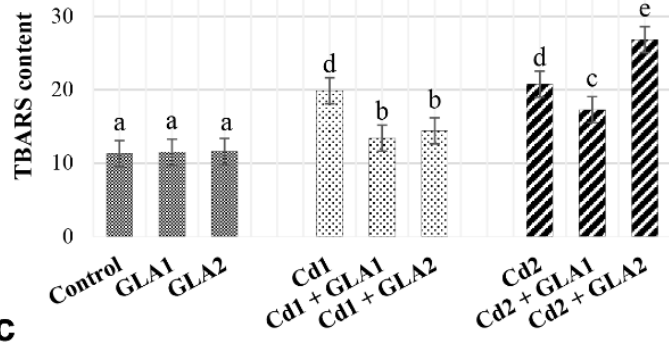

Figure 2. Hydrogen peroxide $\left(\mathrm{H}_{2} \mathrm{O}_{2}\right.$, a), the scavenging activity of hydroxyl radical $\left(\mathrm{OH}^{\bullet}, \mathrm{b}\right)$ and TBARS content (c) in wheat roots after $\mathrm{Cd}(\mathrm{Cd} 1,100$ and $\mathrm{Cd} 2,200 \mu \mathrm{M})$ and GLA (GLA1, $25 \mu \mathrm{M}$ and GLA2, $75 \mu \mathrm{M}$ ).

\subsection{The activities of SOD and NOX}

To conversion of superoxide anion radical to $\mathrm{H}_{2} \mathrm{O}_{2}$, SOD has important role as a scavenger under stress [2]. Four bands for SOD isozymes, as called one Mn-SOD and three $\mathrm{Cu} / \mathrm{Zn}$-SOD1-3, were detected throughout the experimental period (Figure 3a). The wheat roots had higher SOD activity under Cd stress and this result was due to intensities of Mn-SOD and $\mathrm{Cu} / \mathrm{Zn}-\mathrm{SOD} 1-3$. The findings of the current experiment were in line with the 
results of Pan et al. [15]. Also, this induction in SOD activity was maintained by GLA application. The isozyme analysis of SOD reveled that GLA application exposed to Cd-treated wheat roots resulted in the enhancement intensities of Mn-SOD and Cu/Zn-SOD2. Our observation is in agreement with earlier reports given by Bhardwaj et al. [16] who showed GLA caused an increment in SOD under drought stress. GLA under $\mathrm{Cd}$ stress did not occur an increase in NOX activity for $7 \mathrm{~d}$. This observation is parallel with results reported by Yildiztugay et al. [9]. However, there was a markedly increase only in GLA2+Cd2 group for NOX activity of wheat roots as compared to the stress alone. This result could be linked with the increased $\mathrm{H}_{2} \mathrm{O}_{2}$ in this group, because $\mathrm{NOX}$ is the other source of $\mathrm{H}_{2} \mathrm{O}_{2}$ [17]. Only 75 $\mu \mathrm{M}$ GLA alone significantly increased NOX activity (Figure 3b).

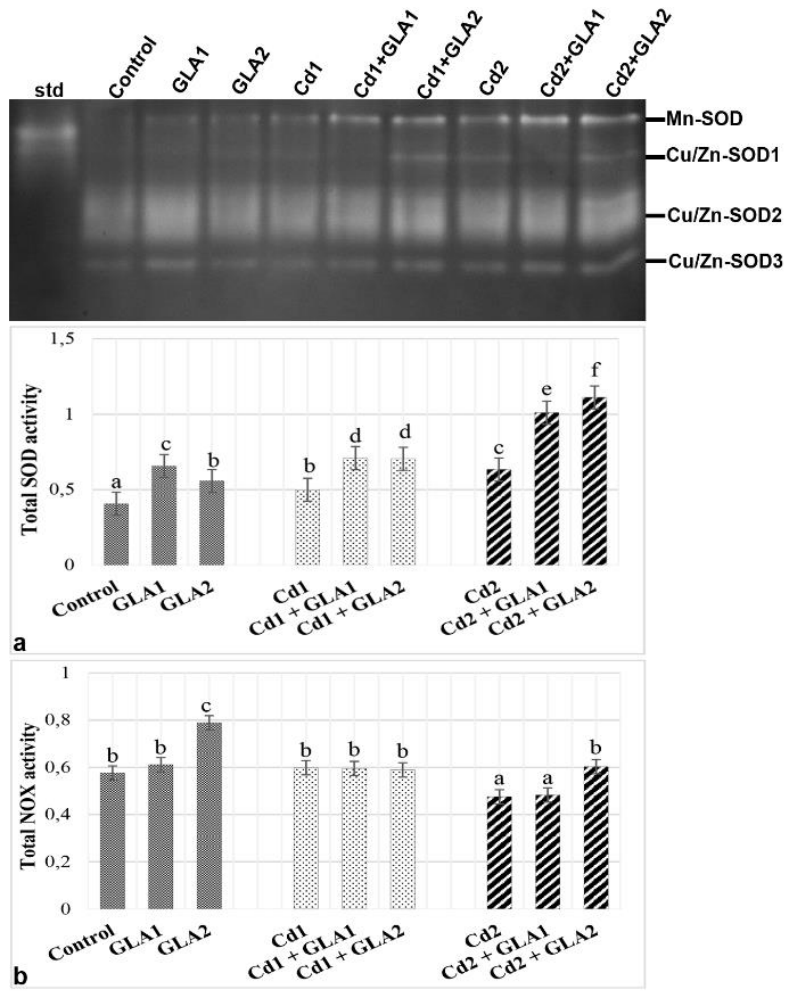

Figure 3. Relative isoenzyme bands and total activity of superoxide dismutase (SOD, a) and total activity of NADPH oxidase (NOX, b) in wheat roots after $\mathrm{Cd}(\mathrm{Cd} 1$, 100 and $\mathrm{Cd} 2,200 \mu \mathrm{M}$ ) and GLA (GLA1, $25 \mu \mathrm{M}$ and GLA2, $75 \mu \mathrm{M})$.

\subsection{The activities of CAT and POX}

As presented in Figure 4a, six CAT isoenzyme bands, CAT1-6, were analyzed in wheat roots. In the presence of $\mathrm{Cd}$ stress, wheat roots showed 7- and 2.1-fold induction in CAT activity, respectively. Similarly, in this group, the band intensity of CAT5 isozyme was stronger than control group. This is supported by Guo et al. [14], who described that stress significantly increased CAT activity. This remarkable increase in CAT was continued by both GLA concentrations under stress, as observed the increased isoforms of CAT2-3-5. Similarly, Bhardwaj et al. [16] concluded that GLA led to upregulation of CAT in wheat.
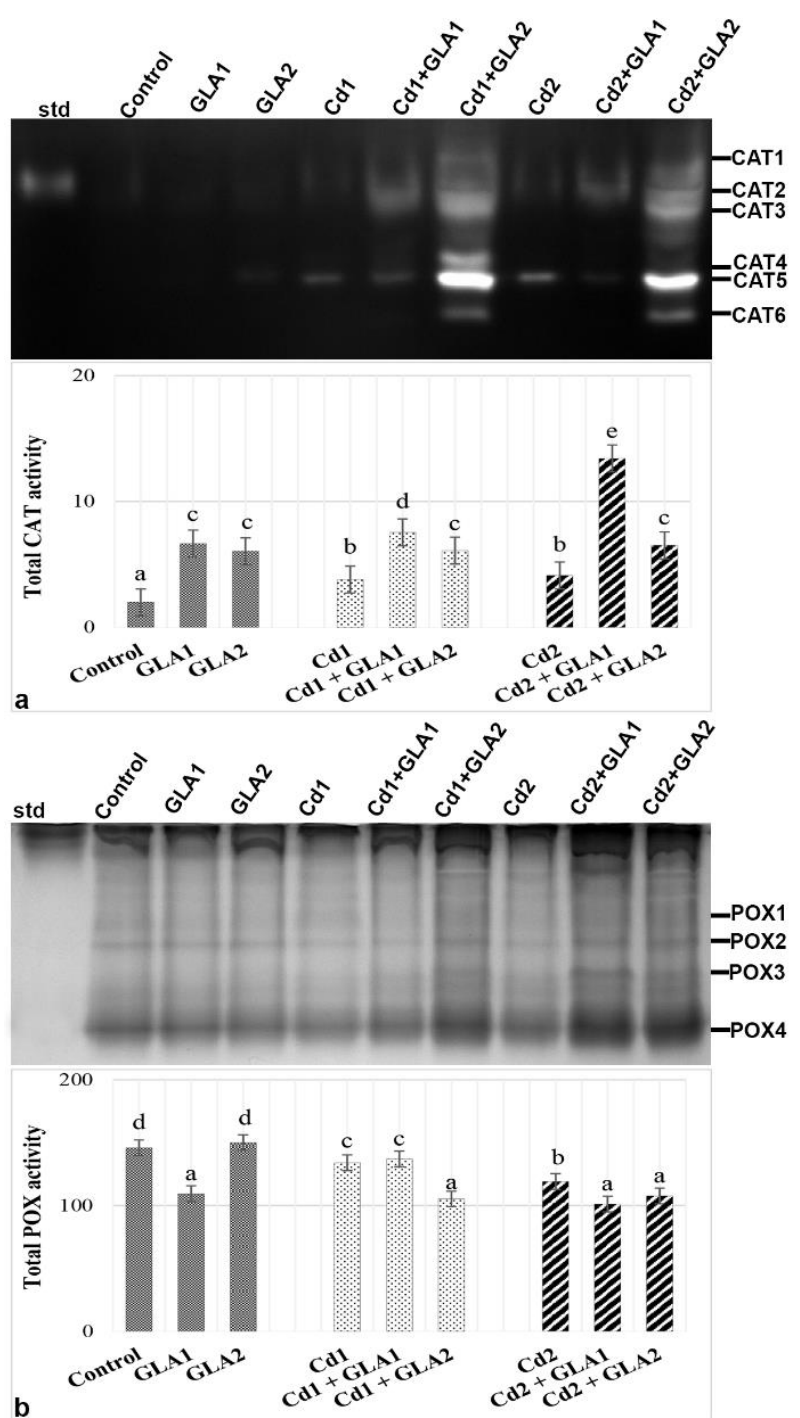

Figure 4. Relative isoenzyme bands and total activity of catalase (CAT, a) and peroxidase (POX, b) in wheat roots after $\mathrm{Cd}(\mathrm{Cd} 1,100$ and $\mathrm{Cd} 2,200 \mu \mathrm{M})$ and GLA (GLA1, $25 \mu \mathrm{M}$ and GLA2, $75 \mu \mathrm{M})$.

Gel analysis revealed that POX isozymes were identified as four bands in wheat roots (Figure 4b). Under stress or stress plus GLA groups, POX activity unchanged or lower than $\mathrm{Cd}$ stress alone. The opposite response was noted by El-Soud et al. [5] that the application of ellagic acid caused an increase in POX activity under osmotic stress. Besides, GLA treatment alone did not occur an increase in POX activity, as providing the non-changed intensity of all POX isozymes.

\subsection{The enzyme/non-enzymes related to AsA-GSH cycle}

As shown in Figure 5a, in treatment groups, three APX isozymes (APX1-3) were identified. The total activity of APX statically observed any substantial effect in wheat 
roots exposed to $\mathrm{Cd}$ stress and the combination form of stress and GLA, except for Cd2+GLA2 which detected a decrease in APX activity (Figure 5a). The data of total APX activity was compatible with the intensities of APX isoforms. In contrast to this, Bhardwaj et al. [16] reported that the upregulation of APX observed under the both GLA and GLA+drought stress. However, GLA application alone triggered an enhancement in APX during the experimental period.
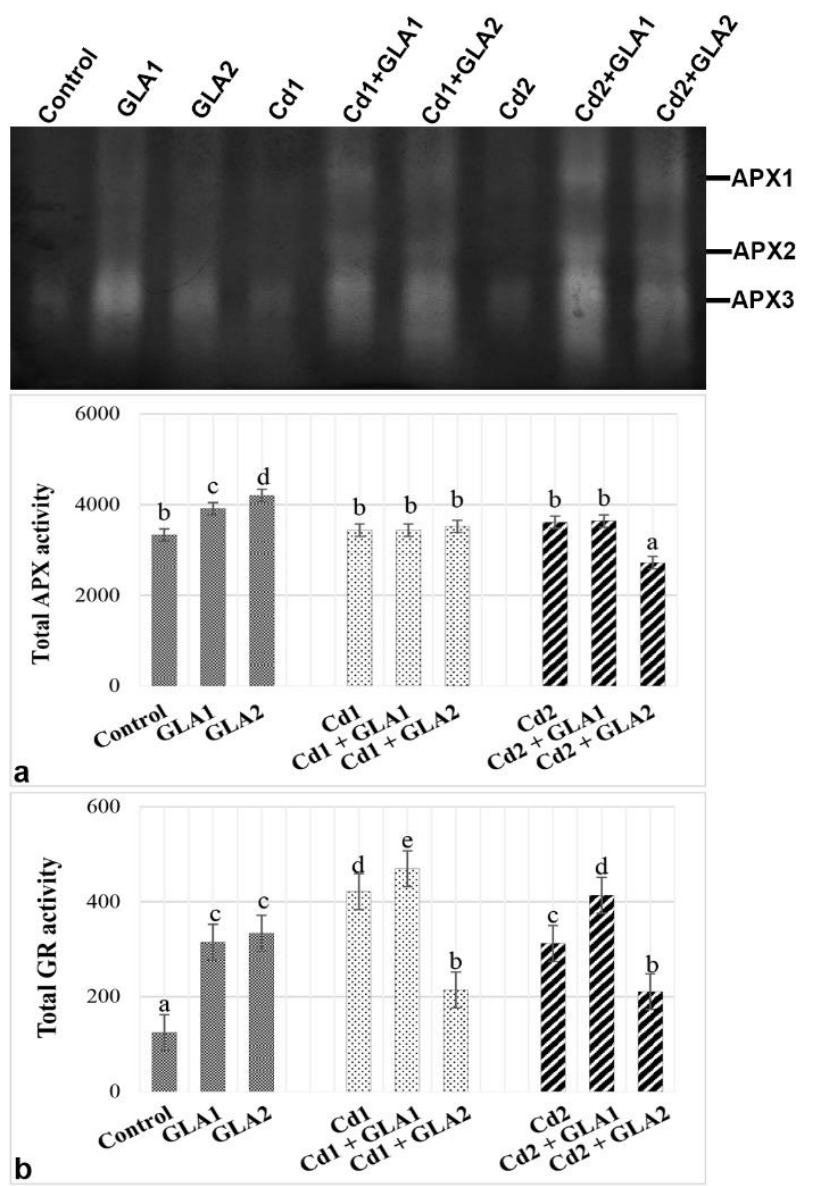

Figure 5. Relative isoenzyme bands and the activity of ascorbate peroxidase activity (APX, a) and glutathione reductase $(\mathrm{GR}, \mathrm{b})$ in wheat roots after $\mathrm{Cd}(\mathrm{Cd} 1,100$ and $\mathrm{Cd} 2,200 \mu \mathrm{M})$ and GLA (GLA1, $25 \mu \mathrm{M}$ and GLA2, 75 $\mu \mathrm{M})$.

On the other hand, under stress conditions, there was an augmentation in total GR activity and this increase was advanced with $100 \mu \mathrm{M} \mathrm{Cd}$ treatment by 3.3-fold (Figure $5 b)$. This finding is in accordance with Rahman et al [18]. The improvement in GR activity was maintained only at GLA1 under the both Cd concentrations. Li et al. [19] showed that after the combination treatments of aminolevulinic acid and PEG-induced drought stress GR activity was induced in cucumber leaves. Similarly, a notable increase in GR activity was in GLA alone-treated roots by 2.5- and 2.6-fold, respectively.

In terms of the activities of MDHAR and DHAR, wheat roots were showed the similar responses. No increase in DHAR (Figure 6a) and MDHAR (Figure 6b) activities of wheat roots treated with GLA alone, stress and their combined groups did not appear during $7 \mathrm{~d}$. However, another study concluded that the cinnamic acid treatment under stress was induced the activities of MDHAR and DHAR [20].

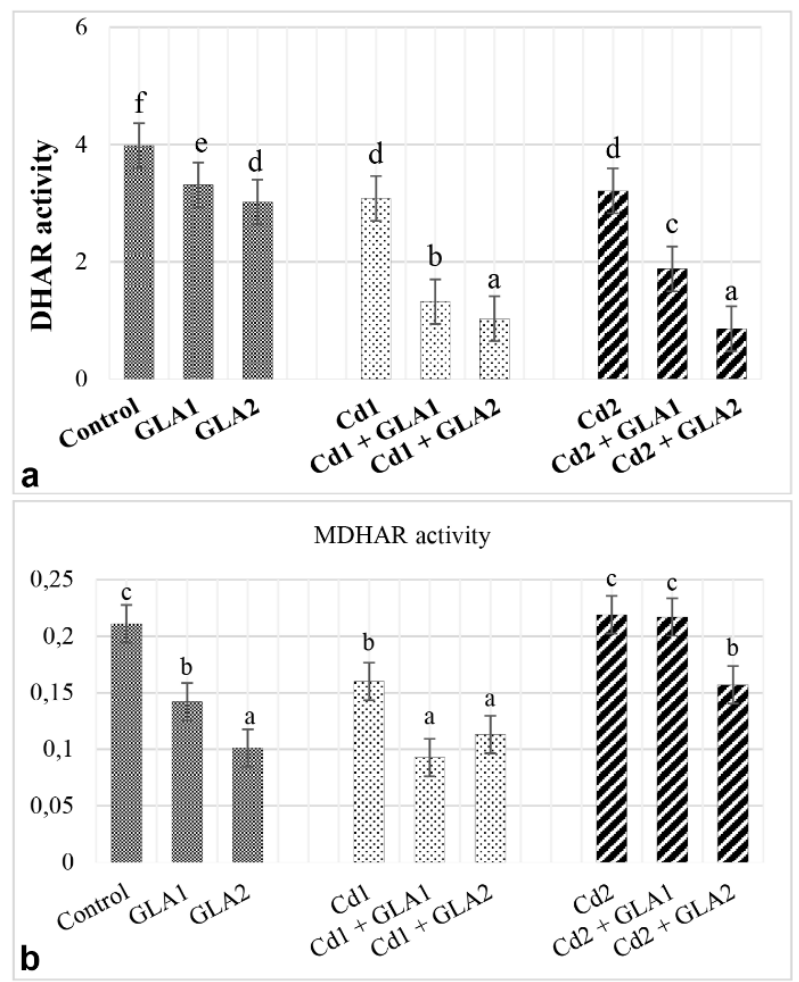

Figure 6. Dehydroascorbate reductase activity (DHAR, a) and monodehydroascorbate reductase activity (MDHAR, b), in in wheat roots after $\mathrm{Cd}(\mathrm{Cd} 1,100$ and $\mathrm{Cd} 2,200 \mu \mathrm{M})$ and GLA (GLA1, $25 \mu \mathrm{M}$ and GLA2, 75 $\mu \mathrm{M})$.

After stress treatments, the contents of GSH (Figure 7a) and GSSG decreased (Figure 7b). Rahman et al. [18] identified a decline in contents of GSH and GSSG in Mntreated plants for $3 \mathrm{~d}$, as similar to the observed findings in the present study. Also, GLA could not increase the contents of GSH and GSSG and the regeneration of GSH under stress conditions. Therefore, GSH/GSSG of plants exposed to GLA+Cd was similar to the stress alone. This was incompatible with earlier report given by $\mathrm{Li}$ et al. [19] who showed that 5-aminolevulinic acid caused an enhancement in GSH content in cucumber as compared to PEG stress. It is reported in the other study that the increased ratio of $\mathrm{GSH} / \mathrm{GSSG}$ is related to eliminate damages produced by Cd stress [20]. 


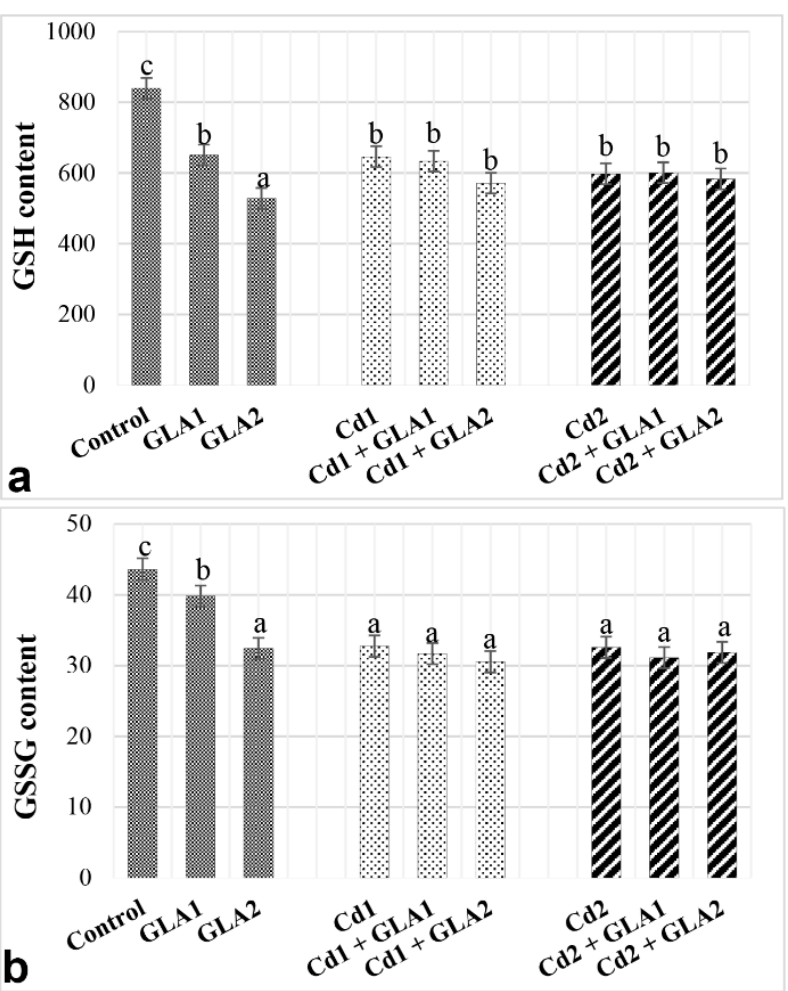

Figure 7. Reduced glutathione content (GSH, a) and oxidized GSH content (GSSG, b) in wheat roots after Cd $(\mathrm{Cd} 1,100$ and $\mathrm{Cd} 2,200 \mu \mathrm{M})$ and GLA (GLA1, $25 \mu \mathrm{M}$ and GLA2, $75 \mu \mathrm{M})$.

\section{Conclusion}

Data of the current study indicate that Cd stress caused the negative impress on growth, Pro content and osmotic potential. However, these parameters were improved by GLA application. GLA2 under the high Cd concentration could not sustain the positive effects on Pro and $\Psi_{\Pi} \mathrm{Cd}$ stress also triggered ROS accumulation and only the activities of SOD, CAT and GR tried to dispose of toxic ROS levels. However, the wheat roots were not successful to eliminate of ROS as evident by increased TBARS content and decreased scavenging activity of $\mathrm{OH}^{\bullet}$. Exogenously applied GLA could activate the first step of defense system (SOD activity) against Cd stress. $\mathrm{H}_{2} \mathrm{O}_{2}$ content produced by SOD could effectively scavenge increased CAT activity at GLA+Cd. This protective effect was also observed in plants with both GLA concentrations under $\mathrm{Cd}$ concentrations. However, to remove of $\mathrm{H}_{2} \mathrm{O}_{2}$ content, GLA did not induce the enzymes and non-enzymes (the activities of GR, APX, DHAR, MDHAR and, the contents of GSH and GSSG) related to AsA-GSH cycle. Under high Cd concentration, despite of increased CAT activity, GLA2 could not eliminate $\mathrm{H}_{2} \mathrm{O}_{2}$ content because of increased NOX activity and then in this group (Cd2+GLA2) the scavenging capacity of $\mathrm{OH}^{\bullet}$ did not change and TBARS content increased.

\section{Author's Contributions}

Ceyda Ozfidan-Konakci: Design of the experimental, Drafted and wrote the manuscript, performed the experiment and result analysis.

\section{Ethics}

There are no ethical issues after the publication of this manuscript.

\section{References}

1. Asgher, M, Khan, NA, Khan, MIR, Fatma, M, Masood A. 2014. Ethylene production is associated with a-lleviation of cadmiuminduced oxidative stress by sulfur in mustard types differing in ethylene sensitivity. Ecotoxicology and Environmental Safety; 106: 54-61.

2. Roychoudhury, A, Basu, S, Sengupta, DN. 2012. Antioxidants and stress-related metabolites in the seedlings of two indica rice varieties exposed to cadmium chloride toxicity. Acta Physiologica Plantarum; 34: 835-847.

3. Srivastava, AK, Srivastava, S, Mishra, S, Prasanna, P, D’Souza, SF. 2014. Identification of redox-regulated components of arsenate (AsV) tolerance through thiourea supplementation in rice. Metallomics; 6: 1718-1730.

4. Kim, Y, Mun, BG, Wagas, M, Kim HH, Shahzad, R, Imran, M, Yun, BW, Lee, IJ. 2018. Regulation of reactive oxygen and nitrogen species by salicylic acid in rice plants under salinity stress conditions. Plos one; 13(3): 1-20.

5. El-Soud, WA, Hegab, MM, AbdElgawad, H, Zinta, G, Asard, H. 2013. Ability of ellagic acid to alleviate osmotic stress on chickpea seedlings. Plant Physiology Biochemistry; 271: 173-183.

6. Michalak, A. 2006. Phenolic compounds and their antioxidant activity in plants growing under heavy metal stress. Polish Journal of Environmental Studies; 15: 523-530.

7. Kamdem, JP, Stefanello, ST, Boligon, AA, Wagner, C, Kade, IJ, Pereira, RP, Preste, AD, Roos, DH, Waczuk, EP, Appel, AS, Athayde, Ml, Souza, DO, Rocha. JBT. 2012. In vitro antioxidant activity of stem bark of Trichilia catigua Adr. Juss. Acta Pharmaceutica; 62: 371-382.

8. Ozfidan-Konakci, C., Yildiztugay, E., Kucukoduk, M. 2015. Protective roles of exogenously applied gallic acid in Oryza sativa subjected to salt andosmotic stresses: effects on the total antioxidant capacity. Plant Growth Regulation; 75(1): 219-234.

9. Yildiztugay, E, Ozfidan-Konakci, C, Kucukoduk, M. 2017. Improvement of cold stress resistance via free radical scavenging ability and promoted water status and photosynthetic capacity of gallic acid in soybean leaves. Journal of Soil Science and Plant Nutrition; 17 (2): 366-384.

10. Breiman, A, Graur, B. 1995. Wheat Evolution. Israel Journal of Plant Science; 43: 85-98.

11. Singh, R, Parihar, P, Singh, M, Bajguz, A, Kumar, J, Singh, S, Singh, VP, Prasad, SM. 2017. Uncovering potential applications of Cyanobacteria and algal metabolites in biology, agriculture and medicine: current status and future prospects. Frontiers in Microbiology; 8, 515.

12. Hussain, MI, Gonzalez, L, Reigosa, MJ. 2011. Allelopathic potential of Acacia melanoxylon $\mathrm{R}$. Br. on the germination and root growth of native species. Weed Biolology and Management; 11: $18-28$. 
13. Mattioli, R., Falasca, G., Sabatini, S., Altamura, M.M., Costantino, P., Trovato, M. 2009. The proline biosynthetic genes P5CS1 and P5CS2 play overlapping roles in Arabidopsis flower transition but not in embryo development. Physiologia Plantarum; 137, 72-85.

14. Guo, J, Shiyu, Q, Rengel, Z, Gao, W, Nie, Z, Hongen, L, Chang, L, Zhoa, P. 2019. Cadmium stress increases antioxidant enzyme activities and decreases endogenous hormone concentrations more in Cd-tolerant than Cd-sensitive wheat varieties. Ecotoxicology and Environmental Safety; 172: 380-387.

15. Pan, C, Haoliang, L, Jinfeng, Y, Yumei, L, Chonling, Y. 2019 Identification of Cadmium-responsive Kandelia obovata SOD family genes and response to $\mathrm{Cd}$ toxicity. Environmental and Experimental Botany; 162: 230-238.

16. Bhardwaj, R, Kaur, L, Srivastava, P. 2017. Comparative Evaluation of Different Phenolic Acids as Priming Agents for Mitigating Drought Stress in Wheat Seedlings. Proceedings of the National Academy of Sciences, India Section B: Biological Sciences; 87(4): 1133-1142.

17. Keunen, E, Peshev, D, Vangronsveld, J, Van Den Ende, W Cuypers, A. 2013. Plant sugars are crucial players in the oxidative challenge during abiotic stress: extending the traditional concept. Plant Cell and Environment; 36: 1242-1255.

18. Rahman, A, Hossain, S, Mahmud, J, Nahar, K, Hasanuzzaman, M Fujita, M. 2016. Manganese-induced salt stress tolerance in rice seedlings: regulation of ion homeostasis, antioxidant defense and glyoxalase systems. Physiology and Molecular Biology of Plants; 22(3): 291-306.

19. Li, Q., Yu, B., Gao, Y., Dai, A.H., Bai, J.G. 2011. Cinnamic acid pretreatment mitigates chilling stress of cucumber leaves through altering antioxidant enzyme activity. Journal of Plant Physiology; 168: 927-934.

20. Dai, A.H., Nie, Y.X., Yu, B., Li, Q., Lu, L.Y., Bai, J.G. 2012 Cinnamic acid pretreatment enhances heat tolerance of cucumber leaves through modulating antioxidant enzyme activity. Environmental and Experimental Botany; 79: 1-10. 